\title{
Knee Surgery and Vascular Lesion: Cases Series and Literature Review
}

\author{
Stefano Rigotti ${ }^{1}$, Luca Garriboli ${ }^{2}$, Gianluca Piovan${ }^{1}$, Daniele Screpis ${ }^{1}$, \\ Antonio Jannello ${ }^{2}$, Claudio Zorzi ${ }^{1}$ \\ ${ }^{1}$ Orthopaedics and Traumatology Department, Ospedale Sacro Cuore Negrar, Verona, Italy \\ ${ }^{2}$ Vascolar Surgery Department, Ospedale Sacro Cuore Negrar, Verona, Italy \\ Email: stefano.rigotti@sacrocuore.it
}

Received 26 August 2014; revised 3 October 2014; accepted 5 November 2014

Copyright (C) 2014 by authors and OALib.

This work is licensed under the Creative Commons Attribution International License (CC BY).

http://creativecommons.org/licenses/by/4.0/

(c)

\begin{abstract}
Iatrogenic vascular injuries to the knee in orthopedic surgery are a major concern for surgeons and a major problem for patients. They are fortunately rare, but it is equally difficult to process. The first decisions taken as soon as you confirm the diagnosis are the most important for the patient's recovery. We did a review of the cases in our center where they are present simultaneously in the Department of Orthopaedic Surgery and Vascular Surgery.
\end{abstract}

\section{Keywords}

Knee Arthroplsty, Vascular Lesion, Knee Surgery, Vascular Complication

Subject Areas: Emergency \& Critical Care, Orthopedics

\section{Introduction}

Knee surgery may involve vascular complications during the arthroplasty, but also with arthroscopic surgery, as well as with the femoral or tibial osteotomy. Vascolar lesion could be involved arterial a venus, but normally the arterial injuries need an immediate treatment, despite venus often could be sub-clinical and recognized in postoperative time. Complications could be acute with severe intraoperative morbidity and significant risk of lower limb amputation, and later with thrombosis, pseudo-aneurysm and arteriovenous fistula. The causes of arterial damage include joint manipulation and dislocation, direct trauma using posterior retractor, k-wire and oscillating saw, as well as the abuse of thigh tourniquet in patients with pre-existing arteriopathy.

Despite the frequency of damages is low, the severity of the clinical outcome necessitates immediaten action by experts in vascular surgery.

In this series we report the experience of our orthopedic center in conjunction with the department of vascular

How to cite this paper: Rigotti, S., Garriboli, L., Piovan, G., Screpis, D., Jannello, A. and Zorzi, C. (2014) Knee Surgery and Vascular Lesion: Cases Series and Literature Review. Open Access Library Journal, 1: e1096.

http://dx.doi.org/10.4236/oalib.1101096 
surgery for popliteal artery damages after 8 years of cooperation, and more than 7000 knee surgeries.

\section{Patients and Methods}

Between 2006 and 2013 in our Institute, 7150 patient underwent knee surgery for ligament repair or total knee arthroplasty (TKA). During this period six popliteal arteries were damaged; the patients were four men and women, the average age was 54 years $(16$ - 84). In all cases were used a thigh tourniquet and the vascular damage was detected at the end of the procedure in three patients, in two within six hours after surgery, while in 1 case the patient presented calf claudicatio after the discharge and the dissection of the popliteal artery was diagnosed 6 months later.

The popliteal artery injury was caused by the oscillating saw in five cases, in three cases (two intra-operative diagnosed, one with six hours in departement) while performing a total knee arthroplasty, one case (diagnosed after 6 months) a revision prosthesis for periprosthetic fracture, one case (within 6 hours diagnosed) during an proximal tibial osteotomy; the sixth patient (immediatly diagnosed) was a young patient and the injury was caused by a k-wire performing a tibial tunnel during PLC reconstruction.

When the orthopedic surgeon detected the arterial injury he controlled the bleeding first with proximal pneumatic compression and then immediately called the vascular team. Our protocol includes the performing of an ultrasound scan to evaluate the patency of distal vessels and the presence and characteristics of the flow. After that, if arterial damage was suspected, an angiography is performed through the common femoral artery in anterograde approach.

In two cases the vascular repair was performed with direct open surgery, in four cases an endovascular treatment was done. The choice between surgical or endovascular operation, normally depends on the operator's experience and if vascular injury has already been recognized intra-operatively. Direct suture of arterial damage was performed in one surgical case, while the second one was repaired using a vascular patch.

In one patient, the youngest, the endovascular treatment was used as a bridge to control the bleeding and after three months the patient underwent surgical reconstruction of the popliteal artery using the autologous saphenous vein.

Patients that had a stent (cover or not) implanted received double anti-platelet therapy (aspirin $100 \mathrm{mg}$ and clopidogrel $75 \mathrm{mg}$ daily) postoperatively up to three months and after that only one drug (aspirin $100 \mathrm{mg}$ daily) was maintained life-long. Postoperative anticoagulant therapy with low molecular weight heparin was administered only in selected cases.

Surgical patients received a single anti-platelet therapy (aspirin $100 \mathrm{mg}$ day) for three months.

The joint function has been limited with the use of articulated brace with ROM $0^{\circ}-60^{\circ}$ for 1 month; following the increase joint was allowed only after evaluation of the vascular surgeon. lnstead the weight bearing was allowed following the normal routine post-operative care.

\section{Results}

Between 2006 and 2013 in our Institute, 7150 patient underwent knee surgery for arthroscopy or open surgery, only six patients $(0.08 \%)$ had arterial complications, $5(0.07 \%)$ that required emergent vascular surgery and 1 $(0.013 \%)$ required delayed treatment for vascular damage.

The most frequent orthopedic treatment was TKA, this is in agreement with literature that reports imilar data. After vascular treatment all patients were discharged without vascular complications.

No limb loss occurred and the length of the postoperative hospital stay was the same as the uncomplicated orthopedic patients for the endovascular group, while the surgical patients needed to stay longer.

\section{Discussion}

The knee surgery is one of the most frequent in orthopedic practice, the patient are both young and older, normally in the first case as consequence of injuries (sports medicine) and in second for tissue degeneration.

Further the increase in length of life of the older people leads to rise the number of patients that will undergo to revision surgery.

Kim et al. [1] showed in a cadaveric angiographic model that the knee flexion can prevent a damage of the popliteal artery during the knee maneuvers, but not excludes that possibility because in about $20 \%$ of patient the 
knee flexion pushes the artery toward the bone space, furthermore exists the possibility of an aberrant course of the popliteal artery.

Also Smith et al. [2], using magnetic resonance imaging, concluded that knee flexion does not guarantee the safety of the popliteal artery during knee surgery.

For these reasons and despite the arterial damages are rare is important to know that, when a problem occurs, is a dramatic event that can lead to limb loss or worse can be a risk for patient life.

Despite of orthopedich in vascular surgery is normally deal with old patients with an atherosclerotic vascular tree. By the time the vascular system is able to increase his collateral ways and, when an occlusion occurs, the distal vascular bed appears preserved in angiographic images. So is therefore recommended to perform a preoperative vascular assessment in elderly patients, especially those who have to undergo revision surgery prosthetics; so it is possible to prevent complications.

The first step for an orthopedic surgeon is to distinguish an arterial or vascular bleeding. If the bleeding starts when the tourniquet is still tension is likely to be of venous origin (from the peripheral circulation of the leg), assuming that the bleeding occurs just dumped the tourniquet is more likely to be of arterial origin. In the latter case is considered a surgical emergency, must be sought the peripheral arterial pulse, possibly located the vascol lesion, alerted the vascular surgeon and repositioned the tourniquet. After a total knee replacement becomes difficult to assess the popliteal space for prosthetic components, so it is useful, if it is allowed by the type prosthesis, to insert the polyethylene component only after releases the tourniquet and excluded the bleeding; this maneuver should be considered routinely when performing prosthetic revision surgery. When it is difficult to detect direct vascular injury is essential to evaluated with an eco-fast track that can be performed directly by the vascular surgeon and it is useful that orthopedic surgeons, who perform surgery of the knee in the absence of a vascular support, have a familiarity with ultrasound emergency methods, because even if the incidence of injury is low morbidity of this lesion vascular is high.

\section{Conclusion}

In conclusion these are the key points when we deal with an emergency: The knowledge of the radiological vascular anatomy; the availability of the endovascular materials (direct suture or endovascol stent); and most important, the habit to think quickly. Calligaro [3] affirmed that patients with a revision prosthetic surgery have twice risk to have a arterial vascular lesion compared to patients with first total knee replacement; in our experience only one (16\%) was a redo-patient. We have identified a trick in emergency to detect if there is a peripheral vascularity: to place the pulse oximeter to the first toe of the limb with suspected lesion. This maneuver allows assessing the flow from the popliteal artery. The positioning of the pulse oximeter on the fifth toe gives us information about the blood from the posterior tibial artery and the interosseous vessels.

\section{References}

[1] Kim, J., Allaire, R. and Harner, C.D. (2010) Vascular Safety during High Tibial Osteotomy: A Cadaveric Angiographic Study. The American Journal of Sports Medicine, 38, 810-815. http://dx.doi.org/10.1177/0363546510363664

[2] Smith, P.N., Gelinas, J., Kennedy, K., Thain, L., Rorabeck, C.H. and Bourne, R.B. (1999) Popliteal Vessels in Knee Surgery. A Magnetic Resonance Imaging Study. Clinical Orthopaedics and Related Research, 367, 158-164.

[3] Calligaro, K.D., Dougherty, M.J., Ryan, S. and Booth, R.E. (2003) Acute Arterial Complications Associated with Total Hip and Knee Arthroplasty. Journal of Vascular Surgery, 38, 1170-1175.

http://dx.doi.org/10.1016/S0741-5214(03)00918-2 